University of Nebraska - Lincoln

DigitalCommons@University of Nebraska - Lincoln

April 1971

\title{
Length and Velocity Formulas in Approximate Oscillator-Strength Calculations
}

Anthony F. Starace

University of Nebraska-Lincoln, astarace1@unl.edu

Follow this and additional works at: https://digitalcommons.unl.edu/physicsstarace

Part of the Physics Commons

Starace, Anthony F., "Length and Velocity Formulas in Approximate Oscillator-Strength Calculations" (1971). Anthony F. Starace Publications. 2.

https://digitalcommons.unl.edu/physicsstarace/2

This Article is brought to you for free and open access by the Research Papers in Physics and Astronomy at DigitalCommons@University of Nebraska - Lincoln. It has been accepted for inclusion in Anthony F. Starace Publications by an authorized administrator of DigitalCommons@University of Nebraska - Lincoln. 


\footnotetext{
${ }^{10}$ D. R. Hartree, The Calculation of Atomic Structures (Wiley, New York, 1957).

${ }^{11} \mathrm{~A}$ complete description of these methods along with flow diagrams and computer listings are given in Ref. 8 .

${ }^{12}$ We have found the initial slope $a_{o}$ to be the most practical criterion for comparing the form of the firstand second-generation integrals.

${ }^{13}$ In practice, the tolerence on normalization is quite loose on the first few potential cycles but is gradually tightened on later cycles.

${ }^{14}$ It is important not to confuse the homogeneous integral, to which corresponds the effective quantum number $n_{H}^{*}$, with the self-consistent integral of the Hartree model; the former is the integral of the homogeneous equation with the HF potential term while the latter is the integral of the homogeneous equation with the Hartree potential term. These potential terms are analytically
}

identical (when $q_{i}=1$ ) but numerically quite different because of the different wave functions used to compute them; therefore, the effective quantum numbers calculated using these two potential terms will undergo drops at different values of $Z$-see Ref. 1, Table II.

${ }^{15}$ It is for this reason that all orbitals involved in groundstate configurations can be readily handled with Hartree's standard methods.

${ }^{16}$ The detailed variation of solution type with $Z$ does depend somewhat on the assumed core-electron configuration.

${ }^{17}$ R. D. Cowan, J. Opt. Soc. Am. $\underline{58}, 924$ (1968).

${ }^{18}$ This behavior of quantum defect is in qualitative agreement with experiment, and is explained in Ref. 1 with the aid of HX effective potentials.

${ }^{19}$ G. W. Pratt, Jr., Phys. Rev. $\underline{88}, 1217$ (1952).

\title{
Length and Velocity Formulas in Approximate Oscillator-Strength Calculations ${ }^{*} \dagger$
}

\author{
Anthony F. Starace \\ Department of Physics, The University of Chicago, Chicago, Illinois 60637 \\ (Received 5 October 1970)
}

\begin{abstract}
The matrix element for electric dipole transitions is correctly given by the length formula in the Hartree-Fock, configuration-interaction, and related approximations, which involve the diagonalization of an approximate, but nonlocal, Hamiltonian.
\end{abstract}

\section{INTRODUCTION}

Fano and Cooper ${ }^{1}$ have emphasized the lack of a consistent theoretical formula for calculating transition matrix elements and their contributions to the Thomas-Reiche-Kuhn sum rule with approximate wave functions. It is generally known that there exist three alternative forms for the electric dipole transition matrix element, called the length, velocity, and acceleration formulas. ${ }^{2}$ These formulas are equivalent when one uses exact eigenfunctions of either the complete spinindependent Hamiltonian or of an independentelectron central-field-model Hamiltonian. However, they disagree whenever the eigenfunctions of a central-field model are improved by including some correlation, even by only the Hartree-Fock procedure. ${ }^{1-5}$ In practice, the disagreement among the alternative formulas is less bothersome than the lack of some criterion to choose among them. Indeed, there are cases ${ }^{3,5}$ in which length and velocity formulas using improved wave functions differ by a factor of 2 and yet either would be more accurate than a central-field-model calculation, in which all alternative formulas give the same result.

This paper identifies a class of approximations in which the length formula is the physically correct one for calculating the transition matrix element for electric dipole processes. This class encompasses all those procedures which solve for the exact eigenfunction of an approximate Hamiltonian. Two examples are the Hartree-Fock and the configuration-interaction approximations. Explicitly excluded from this class are those variational procedures which do not give exact solutions to an approximate Hamiltonian; for these, qualitative considerations must be used to determine which of the alternative formulas are likely to be most accurate. ${ }^{2,6}$ For conciseness we shall compare only the length and velocity formulas.

\section{THEORY}

The difficulty that arises in the interpretation of the length and velocity formulas stems from the occurrence of nonlocal potentials, which is implied by some common approximation procedures, as shown in the next section. Here, therefore, we discuss the properties of nonlocal potentials. Consider first the ordinary case of a local potential $V$. In coordinate representation $V$ is given by $V(\overrightarrow{\mathrm{r}})$, and the effect of operating with $V(\overrightarrow{\mathrm{r}})$ on a wave function $\psi(\vec{r})$ is just the product $V(\vec{r}) \psi(\vec{r})$. However, this is a special case. In general, the 
effect of operating with a potential $V$ on an eigenstate $|\psi\rangle$ is represented by the operator product $V|\psi\rangle$ in coordinate space, $\langle\vec{r}|V| \psi\rangle$; this quantity may be expanded by inserting a complete set of coordinate states between $V$ and $|\psi\rangle$ and summing (or integrating) over this complete set:

$$
\begin{aligned}
\langle\overrightarrow{\mathbf{r}}|V| \psi\rangle & =\int\left\langle\overrightarrow{\mathbf{r}}|V| \overrightarrow{\mathbf{r}}^{\prime}\right\rangle\left\langle\overrightarrow{\mathbf{r}}^{\prime} \mid \psi\right\rangle d^{3} \overrightarrow{\mathbf{r}}^{\prime} \\
& =\int\left\langle\overrightarrow{\mathbf{r}}|V| \overrightarrow{\mathbf{r}}^{\prime}\right\rangle \psi\left(\overrightarrow{\mathbf{r}}^{\prime}\right) \mathrm{d}^{3} \overrightarrow{\mathbf{r}}^{\prime},
\end{aligned}
$$

where $\left\langle\vec{r}^{\prime} \mid \psi\right\rangle$ is the same as the ordinary wave function $\psi\left(\vec{r}^{\prime}\right)$. The difference between local and nonlocal potentials is this. Local potentials are diagonal in coordinate representation, and their matrix elements are given by

$$
\left\langle\overrightarrow{\mathbf{r}}|V| \overrightarrow{\mathbf{r}}^{\prime}\right\rangle=V(\overrightarrow{\mathbf{r}}) \delta\left(\overrightarrow{\mathbf{r}}-\overrightarrow{\mathbf{r}}^{\prime}\right),
$$

since substitution of this expression in the righthand side of Eq. (1) gives the familiar product $V(\vec{r}) \psi(\vec{r})$. Nonlocal potentials are not diagonal in coordinate space and are represented by an integral operator $V\left(\overrightarrow{\mathbf{r}}, \overrightarrow{\mathbf{r}}^{\prime}\right)=\int\left\langle\overrightarrow{\mathbf{r}}|V| \overrightarrow{\mathbf{r}}^{\prime}\right\rangle \cdots d^{3} \overrightarrow{\mathbf{r}}^{\prime}$, which acts on all coordinate functions to its right, as on the right-hand side of Eq. (1). ${ }^{7}$

Two properties of nonlocal potentials are important to this paper. The first is that since a nonlocal potential in coordinate representation is an integral operator, it does not commute with the coordinate operator. The consequence of this is that for Hamiltonians containing nonlocal potentials the length and velocity formulas for the matrix element of an electric dipole transition are no longer equivalent. To see this, take the commutator of the coordinate operator $\sum_{i} \vec{r}_{i}$ with a Hamiltonian $H$ containing a nonlocal potential (atomic units are used throughout this paper):

$$
\sum_{i} \dot{\overrightarrow{\mathrm{r}}}_{i} \equiv i\left[H, \sum_{i} \overrightarrow{\mathrm{r}}_{i}\right]=\sum_{i}\left\{\overrightarrow{\mathrm{p}}_{i}+i\left[V\left(\overrightarrow{\mathrm{r}}_{i}, \overrightarrow{\mathrm{r}}_{i}^{\prime}\right), \overrightarrow{\mathrm{r}}_{i}\right]\right\} \text {. }
$$

Thus, the momentum operator which arises in Eq. (3) from the commutator of the kinetic energy with the coordinates is no longer equivalent to the velocity operator $\sum_{i} \overrightarrow{\mathbf{r}}_{\boldsymbol{i}}$. Taking matrix elements of Eq. (3) between exact eigenstates $\psi_{\alpha}$ and $\psi_{\beta}$ of $H$ (i. e., $H \psi_{\alpha}=E_{\alpha} \psi_{\alpha}$ ) we obtain

$$
\begin{aligned}
& i\left(E_{\alpha}-E_{\beta}\right)\left\langle\psi_{\alpha}\left|\sum_{i} \overrightarrow{\mathrm{r}}_{i}\right| \psi_{\beta}\right\rangle \\
& \quad=\left\langle\psi_{\alpha}\left|\sum_{i} \overrightarrow{\mathrm{p}}_{i}\right| \psi_{\beta}\right\rangle+i\left\langle\psi_{\alpha}\left|\sum_{i}\left[V\left(\overrightarrow{\mathrm{r}}_{i}, \overrightarrow{\mathrm{r}}_{i}^{\prime}\right), \overrightarrow{\mathrm{r}}_{i}\right]\right| \psi_{\beta}\right\rangle .
\end{aligned}
$$

Equation (4) shows that the length formula (on the left-hand side) and the so-called velocity formula (first term on the right-hand side) for an electric dipole transition between states $\alpha$ and $\beta$ differ by the matrix element of the commutator of the nonlocal potential with the coordinates.

A second property of nonlocal potentials eliminates the ambiguity concerning which formula for the transition operator is to be used for Hamilton- ians having nonlocal potentials. This is that nonlocal potentials can be expressed as local momentum-dependent operators, in the following way. Consider the wave function $\psi\left(\overrightarrow{\mathbf{r}}^{\prime}\right)$ on the right-hand side of Eq. (1). We can express the wave function at $\overrightarrow{\mathbf{r}}^{\prime}$ in terms of its value at $\overrightarrow{\mathbf{r}}$ by making a translation by the vector $\overrightarrow{\mathbf{r}}^{\prime}-\overrightarrow{\mathbf{r}}$ :

$$
\psi\left(\overrightarrow{\mathbf{r}}^{\prime}\right)=\left[e^{i\left(\vec{r}^{\prime}-\vec{r}\right) \cdot \vec{p}}\right] \psi(\overrightarrow{\mathbf{r}}),
$$

where the exponential is the translation operator ${ }^{8}$ and $\overrightarrow{\mathrm{p}}=-i \vec{\nabla}_{r}$. Substituting this expression for $\psi\left(\overrightarrow{\mathbf{r}}^{\prime}\right)$ in Eq. (1) we obtain

$$
\begin{aligned}
\int\left\langle\overrightarrow{\mathbf{r}}|V| \overrightarrow{\mathbf{r}}^{\prime}\right\rangle \psi\left(\overrightarrow{\mathbf{r}}^{\prime}\right) \mathrm{d}^{3} r^{\prime} & =\left\{\int\left\langle\overrightarrow{\mathbf{r}}|V| \overrightarrow{\mathbf{r}}^{\prime}\right\rangle e^{i\left(\vec{r}^{\prime}-\overrightarrow{\mathbf{r}}\right) \cdot \overrightarrow{\mathrm{p}}} d^{3} r^{\prime}\right\} \psi(\overrightarrow{\mathbf{r}}) \\
& \equiv V(\overrightarrow{\mathbf{r}}, \overrightarrow{\mathbf{p}}) \psi(\overrightarrow{\mathbf{r}}),
\end{aligned}
$$

where the momentum-dependent form $V(\vec{r}, \vec{p})$ of the nonlocal potential is defined by the expression in curly brackets.

This result alters the interaction of light with particles in a nonlocal potential. Indeed, the interaction Hamiltonian $H_{1 \text { nt }}$ is obtained by making the standard substitution $\overrightarrow{\mathrm{p}}-\overrightarrow{\mathrm{p}}-\overrightarrow{\mathrm{A}}(\overrightarrow{\mathrm{r}}) / c$ [where $\overrightarrow{\mathrm{A}}(\overrightarrow{\mathrm{r}})$ is the vector potential] not only in the kinetic energy operator, but also in $V(\overrightarrow{\mathbf{r}}, \overrightarrow{\mathbf{p}})$. (This procedure is justified by the requirement that one have a gauge-invariant Hamiltonian. ${ }^{9,10}$ ) The additional electromagnetic interaction induced by nonlocal potentials thus arises from the term $V[\overrightarrow{\mathrm{r}}, \overrightarrow{\mathrm{p}}-\overrightarrow{\mathrm{A}}(\overrightarrow{\mathrm{r}}) / c]$ which is given by

$V(\overrightarrow{\mathrm{r}}, \overrightarrow{\mathrm{p}}-\overrightarrow{\mathrm{A}} / c)=\int\left\langle\overrightarrow{\mathrm{r}}|V| \overrightarrow{\mathrm{r}}^{\prime}\right\rangle e^{i\left(\overrightarrow{\mathrm{r}}^{\prime}-\overrightarrow{\mathrm{r}}^{\prime}\right) \cdot\left[\overrightarrow{\mathrm{b}}-\overrightarrow{\mathrm{K}}\left(\vec{r}^{\prime}\right) / c\right]} d^{3} r^{\prime}$.

Treatment of the interaction as a first-order perturbation implies expansion of the exponential in (7) to first order in $\vec{A}$. Limitation to electric dipole transitions implies disregarding the space derivatives of $\vec{A}$ and hence its commutator with $\vec{p}$. Accordingly, Eq. (7) reduces to

$$
\begin{aligned}
V(\overrightarrow{\mathrm{r}}, \overrightarrow{\mathrm{p}}-\overrightarrow{\mathrm{A}} / c)= & \int\left\langle\overrightarrow{\mathrm{r}}|V| \overrightarrow{\mathrm{r}}^{\prime}\right\rangle\left\{1-i\left(\overrightarrow{\mathrm{r}}^{\prime}-\overrightarrow{\mathrm{r}}\right) \cdot \overrightarrow{\mathrm{A}} / c+\cdots\right\} \\
& \times e^{i\left(\overrightarrow{\mathrm{r}}^{\prime}-\overrightarrow{\mathrm{r}}^{\prime}\right) \cdot \overrightarrow{\mathrm{p}}} d^{3} r^{\prime} .
\end{aligned}
$$

Combining the term linear in $\vec{A}$ from Eq. (8) with the usual term arising from the kinetic energy, we obtain for the interaction Hamiltonian

$$
\begin{aligned}
H_{\mathrm{int}}= & -\sum_{i}\left\{\overrightarrow{\mathrm{p}}_{i}\right. \\
& \left.+i \int\left\langle\overrightarrow{\mathrm{r}}_{i}|V| \overrightarrow{\mathrm{r}}_{i}\right\rangle\left(\overrightarrow{\mathrm{r}}_{i}^{\prime}-\overrightarrow{\mathrm{r}}_{i}\right) \cdot d^{3} r_{i}\right\} \cdot \overrightarrow{\mathrm{A}} / c,
\end{aligned}
$$

where we have expressed the term arising from Eq. (8) as an integral operator once again. Noting that this integral operator is just $\left[V\left(\overrightarrow{\mathbf{r}}, \overrightarrow{\mathbf{r}}^{\prime}\right), \overrightarrow{\mathbf{r}}\right]$ and comparing it with Eq. (3), the interaction Hamiltonian becomes simply

$$
H_{1 \mathrm{nt}}=-i\left[H, \sum_{i} \overrightarrow{\mathrm{r}}_{i}\right] \cdot \overrightarrow{\mathrm{A}} / c=-i \sum_{i} \dot{\overrightarrow{\mathrm{r}}}_{i} \cdot \overrightarrow{\mathrm{A}} / c .
$$

Thus, we see that the electrons are coupled to the electromagnetic field through their current, which 
is given by the velocity operator $\sum_{i} \dot{\overrightarrow{\mathrm{r}}}_{i}$ and which only equals the momentum $\sum_{i} \overrightarrow{\mathrm{p}}_{i}$ for local potentials. ${ }^{1}$ The matrix elements of Eq. (10) between exact eigenfunctions of the approximate Hamiltonian $H$ coincide with the length formula, Eq. (4), to within the multiplicative constant $\vec{A} / c$.

\section{EXAMPLES}

Let us now consider some approximation methods which are widely used. We shall see that they involve finding the eigenstates of an approximate Hamiltonian which contains a nonlocal potential. Because of the nonlocal potential, the length and velocity formulas are not equivalent [cf. Eq. (4)]. However, because in each case the exact eigenstates of the approximate Hamiltonian $H$ are found, the transition operator $\sum_{i} \dot{\vec{r}}_{i} \equiv i\left[H, \sum_{i} \overrightarrow{\mathrm{r}}_{i}\right]$ can be evaluated in terms of the length formula.

(i) In the Hartree-Fock approximation the nonlocal potential is the Fock exchange potential, which is given in coordinate representation by

$$
\left\langle\overrightarrow{\mathbf{r}}\left|V_{\text {Fock }}\right| \overrightarrow{\mathrm{r}}^{\prime}\right\rangle=-\sum_{i=1}^{N} \frac{\psi_{i}^{*}\left(\overrightarrow{\mathbf{r}}^{\prime}\right) \psi_{i}(\overrightarrow{\mathrm{r}})}{\left|\overrightarrow{\mathbf{r}}^{\prime}-\overrightarrow{\mathbf{r}}\right|},
$$

where the $\psi_{i}(\vec{r})$ are the $N$ Hartree-Fock orbitals of the ground-state atom or ion. As discussed elsewhere, ${ }^{11,12}$ one can calculate a complete set of one-electron states, all of which are solutions of the same Hartree-Fock Hamiltonian [whose nonlocal part is given by Eq. (11) or by a suitable modification of Eq. (11)]. Hence, as noted by Amusia et al.,$^{3}$ the length formula is the correct one for the matrix element of electric dipole transitions between such Hartree-Fock states.

(ii) Consider now configuration-interaction calculations for obtaining improved initial- and final-state wave functions. ("Configuration interaction" is used here in the sense of Condon and Shortley.) One starts from a complete set of oneelectron eigenstates of some model Hamiltonian $H_{\text {mod }}$ which contains only ordinary local potentials. One then forms a restricted set (not necessarily finite, though usually so) of $\mathrm{N}$-electron Slaterdeterminant wave functions, which represent the configurations to be mixed. These configurations are mixed by matrix elements of the residual interaction $V$ between the Slater determinants under consideration. (Note that $V=H-H_{\text {mod, }}$ where $H$ is the exact spin-independent Hamiltonian.) In more formal language we can form a projection operator $P=\sum_{i \in P}|i\rangle\langle i|$, where each state $|i\rangle$ is a Slater determinant of the restricted set, and we wish to take into account the portion of the residual interaction represented by $P V P$. That $P V P$ is a nonlocal potential is easily seen by taking its matrix elements in coordinate space ${ }^{13}$ :

$$
\left\langle\overrightarrow{\mathbf{r}}|P V P| \overrightarrow{\mathbf{r}}^{\prime}\right\rangle=\sum_{i, j \in P}\langle\overrightarrow{\mathbf{r}} \mid i\rangle\langle i|V| j\rangle\left\langle j \mid \overrightarrow{\mathbf{r}}^{\prime}\right\rangle
$$

$$
=\sum_{i, j \in P} \psi_{i}^{*}(\overrightarrow{\mathrm{r}})\langle i|V| j\rangle \psi_{j}\left(\overrightarrow{\mathrm{r}}^{\prime}\right) .
$$

Note that if the set of states $i$ and $j$ were complete, we could use closure in Eq. (12), i. e., set $P=1$; Eq. (12) would then reduce to the local $\left\langle\vec{r}|V| \vec{r}^{\prime}\right\rangle$.

Configuration-interaction methods utilize in effect the Hamiltonian

$$
H_{\mathrm{CI}}=H_{\text {mod }}+P V P
$$

and its exact eigenfunctions for the calculation of improved transition matrix elements and oscillator strengths. Since $P V P$ is nonlocal, the length formula should be used in these applications.

(iii) Brueckner-Goldstone perturbation theory ${ }^{11,14}$ does not actually calculate exact eigenfunctions of an approximate Hamiltonian. It starts with a complete set of Hartree-Fock wave functions and treats the residual interaction between the exact Hamiltonian and the Hartree-Fock Hamiltonian as a perturbation. Since the zero-order wave functions are derived from a nonlocal potential, the length formula is the consistent one in zero order. In infinite order, the improved wave functions are solutions of the exact Hamiltonian, which has only local potentials, and hence the velocity formula becomes equivalent to the length formula. However, to any finite order of approximation, say the $n$ th, the wave functions solve the exact Hamiltonian to $n$th order, and hence we must expect length and velocity formulas to disagree by effects of $(n+1)$ th order in the residual interaction. Thus the effect of the nonlocal Hartree-Fock potential is removed only to $n$th order and hence the length formula, which is consistent in both zero and infinite order, is presumably consistent for each finite order of the perturbation.

In using model Hamiltonians containing nonlocal potentials, as in the examples above, a word of caution is in order since the Thomas-ReicheKuhn sum rule is not satisfied by their eigenstates. ${ }^{15}$ The density of oscillator strength per unit energy is given by

$$
\frac{\partial f}{\partial E}=\frac{2}{3}\left(E-E_{0}\right)^{-1}\left|\left\langle\psi_{0}\left|\left[H, \Sigma_{i} \vec{r}_{i}\right]\right| \psi_{E}\right\rangle\right|^{2},
$$

where $\psi_{0}$ is the ground-state wave function of the approximate $H$ of energy $E_{0}$, and $\psi_{E}$ is an excitedstate wave function of energy $E$. Equation (14) is formally the same as in the usual case of local potentials except now the commutator of the Hamiltonian with the coordinates has the additional term $\sum_{i}\left[V\left(\vec{r}_{i}, \vec{r}_{i}^{\prime}\right), \vec{r}_{i}\right]$ [Eq. (3)]. Summing the oscillator strength, we obtain ${ }^{9}$

$$
\int \frac{\partial f}{\partial E} d E=N+\frac{1}{3} \sum_{i}\left\langle\psi_{0}\left|\left[\overrightarrow{\mathbf{r}}_{i},\left[V\left(\overrightarrow{\mathbf{r}}_{i}, \overrightarrow{\mathbf{r}}_{i}^{\prime}\right), \overrightarrow{\mathbf{r}}_{i}\right]\right]\right| \psi_{0}\right\rangle,
$$

where $N$, as usual, is the number of electrons in the atom. The second term is an additional oscillator strength induced by nonlocal potentials. 
We may gain insight into the additional induced oscillator strength by considering once more the configuration-interaction Hamiltonian, Eq. (13). In general the projection operator $P$ may be considered as the sum of two projection operators, $P=P_{f}+P_{i}$, where $P_{f}$ and $P_{i}$ are constructed from the subsets of Slater determinants with the same $J$ and parity as the final and ground states, respectively. These subsets, of course, are separately used to obtain improved final and initial states.

Since, in the electric dipole approximation, initial and final states have opposite parity and since $V$ is even under parity, one obtains

$$
P V P=P_{f} V P_{f}+P_{i} V P_{i},
$$

due to the vanishing of $\mathrm{P}_{f} V P_{i}$ and $P_{i} V P_{f}$. Note further that $P_{f}$ and $P_{i}$, by definition, operate on $\psi_{E}$ and $\psi_{0}$ in the following way:

$$
P_{f} \psi_{0}=P_{i} \psi_{E}=0, \quad P_{f} \psi_{E}=\psi_{E}, \quad P_{i} \psi_{0}=\psi_{0} .
$$

Consider now the matrix element of the nonzero commutator, $\left\langle\psi_{0}\left|\left[P V P, \sum_{\alpha} \overrightarrow{\mathbf{r}}_{\alpha}\right]\right| \psi_{E}\right\rangle$, which brings about the additional oscillator strength [cf. Eq. (14)] . Using Eqs. (16) and (17), this matrix element may be expanded as

$$
\begin{aligned}
\left\langle\psi_{0}\left|\left[P V P, \sum_{\alpha} \overrightarrow{\mathrm{r}}_{\alpha}\right]\right| \psi_{E}\right\rangle & =\left\langle\psi_{0}\left|P_{i} V P_{i} \sum_{\alpha} \overrightarrow{\mathrm{r}}_{\alpha}\right| \psi_{E}\right\rangle \\
& -\left\langle\psi_{0}\left|\sum_{\alpha} \overrightarrow{\mathbf{r}}_{\alpha} P_{f} V P_{f}\right| \psi_{E}\right\rangle .
\end{aligned}
$$

Note that if one were to improve only the initial state (i.e., set $P=P_{i}$ ), then only the first term on the right-hand side would appear, while if only the final state were improved (i.e., $P=P_{f}$ ) then only the second term on the right-hand side would appear. Thus the effects of nonlocal potentials may be minimized by judicious simultaneous improvement of both initial and final states rather than improvement of only one. ${ }^{16}$ (The inequivalence of length and velocity formulas when only the final state is improved has already been noted. ${ }^{5}$ ) In other words, the additional oscillator strength arises from the partitioning of the residual interaction and can be minimized if the partitioning is done sensibly.

\section{CONCLUSIONS}

We have shown that the matrix element for electric dipole transitions is correctly given by the length formula for approximation procedures involving a nonlocal Hamiltonian for which exact eigenstates may be calculated. The discussion has centered on two properties of nonlocal potentials, namely, that they do not commute with the coordinate operator, and that they induce an additional interaction with the electromagnetic field (which is also dependent on the commutator of the potential with the coordinate operator). The first property causes the length and velocity formulas to disagree, while the second shows that the length formula is the correct one for electric dipole transition matrix elements. The second property, however, also creates additional oscillator strength, which causes a departure from the Thomas-ReicheKuhn sum rule. It is shown that for configurationinteraction calculations this additional oscillator strength can be minimized if both initial and final states are improved simultaneously. Examples of approximation procedures for which the length formula is the consistent one are the HartreeFock approximation, the configuration-interaction method, and, probably, the Brueckner-Goldstone perturbation theory.

\section{ACKNOWLEDGMENT}

I wish to thank Professor U. Fano for suggesting this problem to me and for providing much valuable advice and encouragement.
*Work supported in part by U.S. Atomic Energy Commission, under Contract No. C00-1674-38.

$\dagger^{\dagger} \mathrm{To}$ be submitted as part of a thesis to the Department of Physics, The University of Chicago, in partial fulfillment of the requirements for the Ph. D. degree.

\$ National Science Foundation Graduate Fellow.

${ }^{1}$ U. Fano and J. W. Cooper, Rev. Mod. Phys. $\underline{40}$, 441 (1968), Secs. 5.4 and 5.5.

${ }^{2}$ S. Chandrasekhar, Astrophys. J. 102, 223 (1945).

${ }^{3}$ M. Ya. Amusia, N. A. Cherepkov, L. V. Chernysheva, and S. I. Sheftel, Phys. Letters 28A, 726 (1969).

${ }^{4}$ E. S. Chang and M. R. C. McDowell, Phys. Rev. 176,126 (1968).

${ }^{5}$ A. F. Starace, Phys. Rev. A 2 , 118 (1970).

${ }^{6}$ R. J. S. Crossley, Advan. At. Mol. Phys. $\underline{5}, 237$ (1969).

${ }^{7}$ Formulas we use in this paper are written for onebody operators for clarity of notation. The extension to $N$-body operators is straightforward.

${ }^{8}$ If the exponential is expanded in a power series, Eq. (5) represents the Taylor-series expansion of $\psi\left(\vec{r}^{\prime}\right)$ about the point $\vec{r}$. Note that $\vec{p}$ never operates on the displacement vector $\left(\overrightarrow{\mathrm{r}}^{\prime}-\overrightarrow{\mathrm{r}}\right)$ in any expansion of the translation operator. See L. D. Landau and E. M. Lifshitz, Quantum Mechanics, 2nd ed. (Addison-Wesley, Reading, Mass., 1965), p. 45.

${ }^{9}$ R. G. Sachs and N. Austern, Phys. Rev. $\underline{81}, 705$ (1951).

${ }^{10}$ A. M. Korolev, Yadern Fiz. 6 , 353 (1967) [Soviet

J. Nucl. Phys. 6,257 (1968)] .

${ }^{11}$ H. P. Kelly, Phys. Rev. 131, 684 (1963); 136, 896 (1964).

${ }^{12}$ M. Ya. Amusia, N. A. Cherepkov, L. V. Chernysheva, and S. I. Sheftel, Zh. Eksperim. i Teor. Fiz. 6, 1897 (1969) [Soviet Phys. JETP 29, 1018 (1969)].

${ }^{13}$ Note that for simplicity we have represented here the coordinates of all $\mathrm{N}$ electrons by the single coordinate $\overrightarrow{\mathrm{r}}$.

${ }^{14} \mathrm{~J}$. Goldstone, Proc. Phys. Soc. (London) A239, 267 (1957).

${ }^{15} \mathrm{~V}$. Fock, Z. Physik 89, 744 (1934).

${ }^{16}$ This prediction, that nonlocal effects can be minimized by simultaneous improvement of both initial and final states, is supported by recent numerical calculations of M. Ya. Amusia, in Proceedings of the Second International Conference on Atomic Physics, Oxford, England, 1970 (unpublished). 